\title{
Propuesta de un sistema de gestión integrada para laboratorios de investigación universitarios ${ }^{*}$
}

\author{
Proposal for an integrated management system \\ for university research laboratories
}

Andrea del Pilar García Péres**

William Javier Cepeda Páez****

Universidad Santo Tomás - ICONTEC

\section{RESUMEN}

Esta investigación define una propuesta de modelo para un sistema de gestión integrada ajustado a las necesidades de los laboratorios de investigación universitarios que permita asegurar resultados confiables, con base en las normas técnicas ISO 9001:2008 E ISO-IEC 17025:2005. Se utilizan varias estrategias para la recolección y procesamiento de información de datos, tales como diagnóstico, observación, encuestas y se hace una posterior triangulación.
El resultado principal de esta investigación es la de proponer un modelo de sistema de gestión integrada para ser empleada por los laboratorios de investigación con el ánimo de facilitar su gestión interna que esté constituido por una serie de requisitos, una ruta para implementarlos y la representación gráfica que relacione las partes que conforman el modelo. Otros resultados obtenidos fueron la introducción de manera exitosa de los principios de un sistema de gestión integrado y la identificación de los componentes articuladores para las partes que constituyen el modelo.

* Artículo de investigación

** Ingeniera Química, Jefe de laboratorio, Escuela de Medicina y Ciencias de la Salud. Universidad del Rosario. Especialista en Administración y Gerencia de Sistemas de la Calidad. Correo electrónico: apgarcia@gmail.com. Autor para correspondencia.

*** Arquitecto. Colegio Nueva Granada. Especialista en Planificación y Administración del Desarrollo Rural. Correo electrónico: arq.javier. cepeda@gmail.com.. 
Palabras clave: ISO 9001, ISO-IEC 17025, laboratorio de investigación, sistema integrado de gestión, sistema de gestión de la calidad.

\section{ABSTRACT}

This research defines a proposed model for an integrated management system model tailored to the needs of university research laboratories, which would ensure reliable results, based on technical standards ISO 9001: 2008 and ISO-IEC 17025: 2005. Several strategies for collecting and processing data information, such as diagnosis, monitoring, and subsequent triangulation surveys are used.

The main result of this research is the proposal of a model for integrated management system as a tool that can be used by research laboratories to facilitate its internal management, consisting of a series of requirements, the route to implement and a graphical representation that relates the parts that make up the model. Other results reveal the successfully introduction of the principles of an integrated management system and identification of the articulating component parts that make up the model.

Keywords: ISO 9001, ISO 17025, integrated management system, research laboratory, quality management system.

\section{INTRODUCCIÓN}

Se reconoce que es necesario promover la calidad en la investigación involucrando aspectos que permitan asegurar las buenas prácticas en el contexto de los laboratorios de investigación ${ }^{1}$, y garantizando la calidad

1 RESPONSIBLE CONDUCT IN THE GLOBAL RESEARCH ENTERPRISE [en línea]. a policy report. interacademy council. de los métodos empleados por los investigadores para obtener sus resultados².

Aunque actualmente existen varias normas que definen sistemas de gestión que pueden ser implementadas dentro de los laboratorios, tanto en laboratorios del sector público y privado, como en instituciones de investigación y universidades, se ha cuestionado su aplicación por los altos costos, por la demanda de tiempo que interfiere con el uso para las actividades de investigación ${ }^{3}$. Así mismo, por su efecto negativo sobre la libertad de los investigadores, lo cual puede entorpecer su creatividad, y además se cuestiona si estas normas pueden soportar la credibilidad de los resultados investigativos ${ }^{4}$.

Las normas de gestión disponibles para ser aplicadas en los laboratorios de investigación se perciben como elementos que presentan límites en su aplicabilidad y difícil adaptación a procesos no rutinarios, propios de la actividad investigativa como la que se desarrolla en los laboratorios ${ }^{5}$ (Tabla 1). Aunque existen normas que pueden ser aplicadas para la gestión de

septiembre 2012 [consultado el 01 de marzo de 2013]. disponible en: www.interacademycouncil.net. p.15.

2 ALONSO MIGUEL, Pedro. Calidad en Investigación ( $1^{\mathrm{a}}$ y $2^{\mathrm{a}}$ parte). De qué trata la gestión de calidad en investigación. Aula Abierta [en línea]. Diciembre, 2005 [consultado el 09 de Julio de 2012]. No. 32 y 33. Disponible en: http://www.madrimasd.org/revista/ revista32/aula/aula1.asp

3 ROBINS, Margaret M. SCARLL, Jane S. y E. KEY, Pauline. Quality assurance in research laboratories. Accred Qual Assur [en línea]. Springer-Verlag. Abril de 2006, p. 214 - 223. Disponible en: http:// www.springerlink.com/?MUD=MP

4 GESTIÓN DE CALIDAD EN LA INVESTIGACIÓN [en línea]. Red iris [consultado el 01 de Marzo de 2013]. Disponible en: http:// www.rediris.es/list/info/calidadinv.html

5 ROBINS, Margaret M. SCARLL, Jane S. y E. KEY, Pauline. Quality assurance in research laboratories. Accred Qual Assur [en línea]. Springer-Verlag. Abril, 2006. p. 214 - 223. Disponible en: http:// www.springerlink.com/?MUD=MP 
procesos de investigación, estas se enfocan hacia el diseño y desarrollo tecnológico, enmarcadas en la administración de recursos y tareas, obviando aspectos importantes como el enfoque al cliente (el investigador y su investigación) y la gestión del riesgo para las actividades investigativas ${ }^{6}$.

Tabla 1. Problemas para la aplicación de normas en laboratorios de investigación

\begin{tabular}{|c|c|}
\hline NORMA & PROBLEMA \\
\hline ISO 9001 & $\begin{array}{l}\text { Los resultados que se obtienen tras una investigación no necesariamente cumplen con las expectativas del cliente, } \\
\text { ya que estos pueden ser desconocidos o incluso no haber sido planeadas, sin que ello afecte la validez o relevancia } \\
\text { de los resultados obtenidos. }\end{array}$ \\
\hline ISO-IEC 17025 & $\begin{array}{l}\text { Algunos de sus requisitos no se aplican o son muy restrictivos para las rutinas de análisis que se realizan en los } \\
\text { laboratorios de investigación, no describe como registrar u organizar la información relativa a la concepción } \\
\text { experimental, y cómo implementar un ambiente de trabajo favorable a la investigación. }\end{array}$ \\
\hline ISO 15189 & Su aplicabilidad se limita solo a los laboratorios clínicos en el área de salud humana. \\
\hline ISO 10006 & $\begin{array}{l}\text { No incorpora las nociones de suma importancia para las actividades de investigación como son la distinción entre } \\
\text { lo positivo y lo negativo de la falta de conformidad o incluso la noción de no confirmación de hipótesis. }\end{array}$ \\
\hline $\begin{array}{l}\text { ISO 10006, UNE 166001, } \\
\text { FD X } 50\end{array}$ & $\begin{array}{l}\text { Su uso no es muy generalizado y su aplicación se concentra en la administración de recursos y tareas dentro de } \\
\text { los proyectos de investigación, sin contemplar aspectos como la del enfoque al cliente. } \\
\text { No tienen en cuenta que la actividad investigativa se desarrolla de manera diferente en cada región y disciplina. }\end{array}$ \\
\hline
\end{tabular}

Fuente: elaboración propia. Datos tomados de BIRÉ, Ronel; VARAS, Marika y ALONSO MIGUEL, Pedro.

Los aspectos ya descritos no son ajenos para los laboratorios universitarios, los cuales deben enfocarse al cumplimiento de su quehacer como espacios de formación e investigación. Por ello la importancia de plantear un sistema de gestión integrado (SGI) para los laboratorios de investigación universitarios (LIU), que pueda utilizarse como una herramienta para satisfacer las necesidades de las partes interesadas (investigadores, patrocinadores y estudiantes), el cual sea de fácil comprensión e implementación y que permita garantizar la confiabilidad de los resultados obtenidos.

La propuesta del modelo de SGI se estructura bajo tres objetivos: iniciar con un diagnóstico para identificar elementos de gestión que correspondan a requisitos normativos e identificar cuales elementos pueden conformar el modelo, partiendo del supuesto que los LIU cuentan con prácticas que corresponden a exigencias normativas. Realizar el diseño del modelo de SGI basándose en el supuesto de que es posible que un solo modelo integre los elementos de gestión que son requeridos por los LIU. Finalmente, proceder a prevalidar la propuesta verificando si esta puede facilitar a los LIU la confiabilidad necesaria de los resultados generados por los laboratorios.

6 VARAS, Marika. SÁNCHEZ, Luis y ALVARADO, Luis. Los directores de proyectos de investigación: competencias y desafíos. R\&D\&i Project Managers: professional competitions and challenges. Dirección y Organización, Diciembre, 2009 [consultado en Julio 29 de 2012]. No. 39. Disponible en: http://www.revistadyo.com/index. php/dyo/article/viewFile/25/25. ISSN: 1132-175X. 


\section{METODOLOGÍA}

La metodología aplicada para construir la propuesta del modelo se definió teniendo en cuenta que este trabajo es exploratorio y de carácter positivista semicualitativo. Para ello se llevaron a cabo cuatro etapas donde se aplicaron dos métodos: la recopilación de información, y la triangulación para el análisis y procesamiento de la información.

\section{Recolección de información}

Se llevó a cabo una recolección de información preliminar mediante la aplicación de dos instrumentos: una encuesta dirigida a investigadores universitarios y una herramienta de observación para ser aplicada al interior de los LIU, con el propósito de establecer los aspectos relacionados a la gestión integral aplicables a los laboratorios que deberían incluirse en el modelo e identificar cuáles elementos de gestión se implementan por los LIU respectivamente.

Para que los instrumentos aplicados pudieran arrojar resultados que pudieran emplearse dentro de la estructuración del SGI para los LIU se buscó un eje que permitiera la integración y organización de los requisitos normativos tomados de las normas técnicas ISO 9001:2008 "Sistemas de gestión de la calidad. Requisitos" e ISO-IEC 17025:2005 "Requisitos generales para la competencia de laboratorios de ensayo y calibración", ambas aplicables en laboratorios. Se excluyó por su extensión y complejidad el área de salud, es por esto que no se tuvo en cuenta la norma técnica ISO 15189 "Laboratorios Clínicos. Requisitos particulares relativos a la calidad y la competencia" dentro de esta propuesta de modelo.

Los elementos empleados como eje para la estructuración entre las normas ISO 9001 e ISO-IEC 17025 son los principios que fundamentan los sistemas de gestión de calidad descritos dentro de la norma
ISO 9000:2005 "Sistemas de gestión de la calidad. Fundamentos y vocabulario", por ser universales y permitir la integración de las normas seleccionadas como base en un solo sistema de gestión de calidad como se señala en el Joint IAF-ILAC-ISO communiquét.

Los requisitos de las dos normas seleccionadas fueron alineados según su relación con cada principio de la norma técnica ISO 9000; para realizar esto se emplearon categorías y subcategorías derivadas de cada principio, luego se definieron los requisitos más relevantes teniendo en cuenta la problemática de investigación, la mejora continua y los componentes globales de un $\mathrm{SGI}^{8}$ : direccionamiento estratégico, estructura del negocio, sistemas de gestión (normalizados o no) y la cultura de la organización; otro aspecto que se tuvo en cuenta es que un SGI cuenta también con un enfoque de principios equivalentes a los descritos en la norma técnica ISO $9000^{9}$ (Tabla 2).

El contenido de cada instrumento aplicado fue el resultado de la alineación de cada requisito con un principio específico (Figura 1). Tanto la encuesta como la herramienta de observación contemplan todos los principios dentro de su contenido.

7 JOINT IAF-ILAC-ISO COMMUNIQUÉ ON THE MANAGEMENT SYSTEMS REQUIREMENTS OF ISO/IEC 17025:2005 [en línea]. General requirements for the competence of testing and calibration Laboratory. January 2009 IAF, ILAC, ISO [Consultado en Junio 01 de 2013]. Disponible en: http://www.nist.gov/nvlap/ upload/2009-01-08_IAF-ILAG-ISO_communiqu-C3-83-_ISOIEC17025_-1.pdf

8 TEJADA LOSADA, Fabio y PEÑA GUARÍN, Guillermo. Reflexiones sobre las características constitutivas de la gestión integral. SIGNOS. Vol.1, No. 2, 2009. p. 79-93, ISSN 2145-1389.

9 VELÁSQUEZ RUEDA, María del Rosario. Planificación de un sistema de gestión de la calidad como plataforma para integrar otros modelos de gestión. SIGNOS. Vol. 4, No. 2, 2012. p. 15-31. ISSN: 2145-1389. 
Tabla 2. Equivalencia entre principios ISO 9000 y del sistema de gestión integral

\begin{tabular}{|l|l|}
\hline \multicolumn{1}{|c|}{ PRINCIPIO ISO 9000 } & PRINCIPIO BAJO EL ENFOQUE DE GESTIÓN INTEGRAL \\
\hline Enfoque al cliente & Satisfacción de las partes interesadas \\
\hline Liderazgo & Liderazgo \\
\hline Participación del personal & Participación \\
\hline Enfoque basado en procesos & Enfoque por procesos \\
\hline Enfoque de sistema para la gestión & Enfoque de sistema para la gestión \\
\hline Mejora continua & Mejora continua \\
\hline Enfoque basado en hechos para la toma de decisiones & Toma de decisiones basada en un enfoque sistémico \\
\hline Relaciones mutuamente beneficiosas con el proveedor & Relaciones gana-gana con las partes interesadas \\
\hline
\end{tabular}

Fuente: elaboración propia.

Figoura 1. Definición de aspectos a indagar

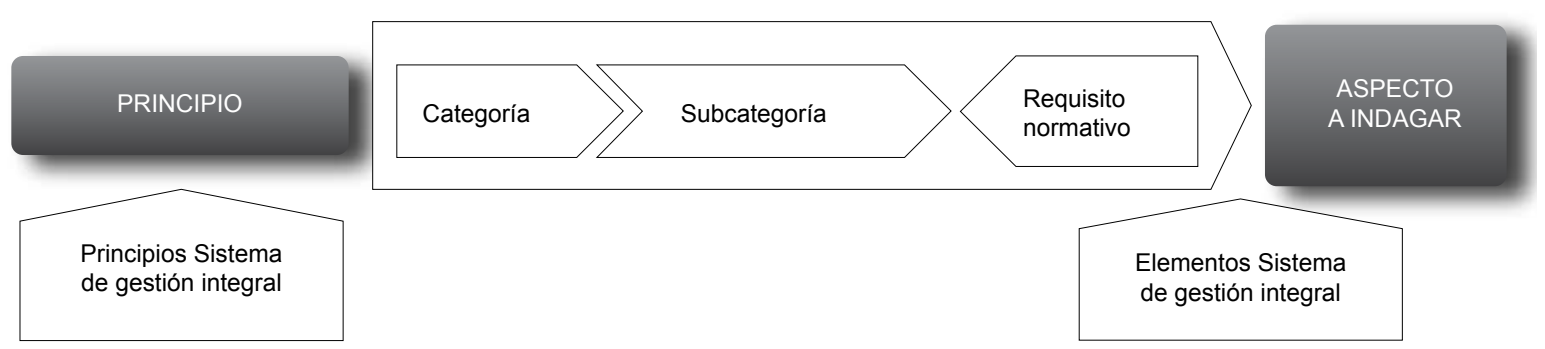

Fuente: elaboración propia.

La muestra utilizada se seleccionó bajo los criterios de un muestreo no probabilístico (por conveniencia), debido al carácter exploratorio de este estudio. Por esta razón los instrumentos fueron aplicados a LIU y a investigadores vinculados con las Universidades Nacional de Colombia y Santo Tomás localizadas en la ciudad de Bogotá (Tabla 3).

Tabla 3. Ficha instrumentos

\begin{tabular}{|c|c|c|c|}
\hline & & ENCUESTA & HERRAMIENTA OBSERVACIÓN \\
\hline \multirow{3}{*}{$\begin{array}{c}\text { TAMAÑO } \\
\text { DE } \\
\text { LA MUESTRA }\end{array}$} & $\begin{array}{l}\text { Universidad Nacional } \\
\text { de Colombia }\end{array}$ & 37 & 11 \\
\hline & Universidad Santo Tomás & 29 & 28 \\
\hline & Total & 66 investigadores & 39 laboratorios \\
\hline \multicolumn{2}{|c|}{ No. DE ASPECTOS DENTRO EL INSTRUMENTO } & 25 afirmaciones & 25 afirmaciones \\
\hline \multicolumn{2}{|c|}{ TIPO DE RESPUESTA } & Escala likert & $\mathrm{Si} / \mathrm{N}_{0}$ \\
\hline \multicolumn{2}{|c|}{ CRITERIO DE CALIFICACIÓN } & $\begin{array}{l}\text { Por puntaje: } \\
4 \text { acepta el elemento } \\
3.9-3 \text { No concluyente } \\
\text { Menor a } 3 \text { se descarta }\end{array}$ & $\begin{array}{l}\text { Se acepta el elemento cuando este } \\
\text { obtiene la mayoría simple }\end{array}$ \\
\hline
\end{tabular}

Fuente: elaboración propia. 


\section{Integración del marco teórico y recopilación de información}

Los resultados de la aplicación de las encuestas, las herramientas de campo y la revisión del marco teórico se integraron mediante triangulación ${ }^{10}$. De este modo, se cruzaron las respuestas obtenidas en cada aspecto indagado para cada instrumento, por subcategoría y categoría, para generar las respectivas conclusiones para el principio de gestión de la calidad. En cada principio se obtuvieron varias conclusiones de acuerdo con el número de categorías definidas al construir los instrumentos de recolección de información. Las conclusiones generales se emplearon luego para estructurar los requisitos que describen el modelo de gestión integral para los LIU.

\section{Construcción del modelo}

Como parte de la construcción del modelo se identificaron tres componentes que permitieron la agrupación e integración de sus partes al mostrar las relaciones entre los elementos del sistema y su entorno, y facilitar la relación entre los principios dentro del sistema ${ }^{11}$, sin afectar la fundamentación realizada a partir de los principios ni el enfoque o contenido de los requisitos definidos. Estos componentes se obtuvieron después de evaluar y comparar los elementos en común entre cuatro enfoques encontrados sobre la gestión al interior de las organizaciones (Tabla 4).

Tabla 4. Enfoques relacionados a la gestión al interior de los procesos

\begin{tabular}{|c|c|c|c|c|}
\hline $\begin{array}{l}\text { FACTORES DE ÉXITO } \\
\text { EN LA GESTIÓN } \\
\text { DE PROCESOS }\end{array}$ & $\begin{array}{l}\text { CADENA DE VALOR } \\
\text { DEFINIDA POR } \\
\text { MICHEL PORTER }\end{array}$ & $\begin{array}{c}\text { NIVELES } \\
\text { DE LA GESTIÓN } \\
\text { ORGANIZACIONAL }\end{array}$ & $\begin{array}{l}\text { ASPECTOS } \\
\text { QUE PERMITEN } \\
\text { LA ARQUITECTURA } \\
\text { ORGANIZACIONAL }\end{array}$ & $\begin{array}{l}\text { COMPONENTE } \\
\text { IDENTIFICADOS } \\
\text { PARA EL MODELO }\end{array}$ \\
\hline Alineación estratégica & Gestión corporativa & Estratégico & -- & Estratégico \\
\hline Gobernanza & -- & -- & -- & -- \\
\hline Cultura organizacional* & -- & -- & -- & \multirow[b]{2}{*}{ Humano** } \\
\hline Personas & $\begin{array}{l}\text { Gestión del recurso } \\
\text { humano }\end{array}$ & \multirow{3}{*}{ Operacional } & Factor humano & \\
\hline Método & $\begin{array}{l}\text { Abastecimiento materias } \\
\text { primas }\end{array}$ & & -- & \multirow{4}{*}{ Operacional } \\
\hline-- & Desarrollo tecnológico & & \multirow[b]{2}{*}{ Factor tecnológico } & \\
\hline Tecnología de la información & -- & -- & & \\
\hline-- & Finanzas y comercialización * & Táctico & -- & \\
\hline \multicolumn{5}{|c|}{$\begin{array}{l}\text { * Se tiene en cuenta al ser parte de los elementos que describen un SGI } \\
\text { ** Se toma como un componente, para los Sistemas de Gestión Integral el factor humano es un aspecto independiente de los demás elementos } \\
\text { que lo componen. }\end{array}$} \\
\hline
\end{tabular}

Fuente: elaboración propia. Tomando como base: HANDBOOK OF BUSINESS PROCESS MANAGEMENT 1. p 45, 57-58, 104.

10 CABRERA CISTERNA, Francisco. Categorización y triangulación como proceso de validación del conocimiento en investigación cualitativa. Ensayo. Theoria, Vol. 14, 2005. p. 61-71. ISSN 0717-196X.
11 ARANGO SERNA, Martín Darío. LONDOÑO SALAZAR, Jesús Enrique y ZAPATA CORTÉS, Julián Andrés. Arquitectura EmpresarialUna visión general. Revista Ingenierías. Universidad de Medellín. Enero-junio, 2010. Vol. 9, No. 16. p. 110-111. ISSN 1692-3324. 
Estos componentes de articulación se emplearon para agrupar los principios según el enfoque presentado por sus correspondientes requisitos como se señala a continuación:

Componente Humano: Involucra a los principios cuyos requisitos están involucrados con la cultura organizacional, las habilidades y conocimientos del personal.

Componente Estratégico: Relaciona los principios cuyos requisitos se relacionan con el direccionamiento al interior de la organización y las relaciones con las partes interesadas.

Componente Operativo: Reúne los principios cuyos requisitos hacen referencia a las actividades que se llevan a cabo y las que están directamente relacionadas con la elaboración del producto o prestación del servicio.

\section{Prevalidación del modelo}

Antes de que el modelo se valide en un LIU se llevó a cabo una evaluación del mismo (prevalidación) por expertos con experiencia en laboratorios de investigación, en actividades de investigación y con conocimientos de las normas ISO 9001 o ISO-IEG 17025. La evaluación cubrió elementos como la facilidad de comprensión de los elementos presentados, la aplicabilidad de los requisitos en los LIU, aspectos relacionados al riesgo de no lograr la confiabilidad de los resultados del laboratorio, el cumplimiento del presupuesto de investigación asignado a las actividades del laboratorio, y además de verificar si el modelo facilita la gestión de conocimiento y aprendizaje dentro del laboratorio.

Para determinar la consistencia interna y validez de la aplicación del instrumento de prevalidación se utilizó el coeficiente de correlación Alfa de
Cronbach ${ }^{12 y 13}$. Los valores del alfa de Cronbach varían entre 0 a 1 y se tomó el valor mínimo de confiabilidad en $0.7^{14}$ por considerase adecuado para definir la consistencia y validez del instrumento empleado para realizar la prevalidación. La estimación de este coeficiente se llevó a cabo mediante un análisis de varianzas ${ }^{15}$.

$\alpha=\frac{\mathrm{k}}{\mathrm{k}-1}\left[1-\frac{\sum_{\mathrm{i}=1}^{\mathrm{k}} \mathrm{S}_{\mathrm{i}}^{2}}{\mathrm{~S}_{\mathrm{t}}^{2}}\right]$

Donde:

$\alpha$ : Coeficiente de Cronbach

k: Número de preguntas realizadas

S2i: Varianza de los resultados de cada pregunta realizada

$\mathrm{S}_{\mathrm{t}}^{2}$ Varianza del total del puntaje dado por cada evaluador (varianza poblacional)

Al obtener un resultado aceptable al estimar el coeficiente de correlación (Tabla 5) se continúa con la revisión de las evaluaciones individuales de cada experto y se realizan los ajustes necesarios al modelo propuesto.

12 GLIEM, Joseph A y GLIEM, Rosemary R [en línea]. Calculating, interpreting, and reporting Cronbach's alpha reliability coefficient for Likert-type scales. Midwest Research-to-Practice Conference in Adult, Continuing, and Community Education. The Ohio State University. October 8-10, 2003 [Consultado el 18 de mayo de 2014]. p. 82-88. Disponible en: https://scholarworks.iupui.edu/ handle/1805/344

13 CERVANTES, Víctor H. [en línea]. Interpretaciones del coeficiente de alpha de Cronbach. Avances en Medición, Universidad Nacional de Colombia. 2005. Vol 3, No. 1. p. 9-28. ISSN 1692-0023. [Consultado el mayo 18 de 2014]. Disponible en: https://scienti. colciencias.gov.co:8084/publidex/docs/artículos/1692-0023/2/1.pdf

14 GLIEM, Joseph A y GLIEM, Rosemary R. Op. Cit. p. 82-88

15 CAMPO ARIAS, Adalberto y OVIEDO C, Heidi [en línea]. Propiedades psicométricas de una escala la consistencia interna. Revista Salud Pública, Universidad Nacional de Colombia. Diciembre, 2008. No. 10, Vol 5. p. 831-839. ISSN 0124-0064. [Consultado el 05 de mayo de 2014]. Disponible en: http://www.scielosp.org/pdf/rsap/v10n5/v10n5a15.pdf 
Tabla 5. Ficha de prevalidación

\begin{tabular}{|c|l|}
\hline TAMAÑO DE MUESTRA & 9 evaluadores \\
\hline DISEÑO MUESTRAL & Por conveniencia \\
\hline $\begin{array}{c}\text { NÚMERO DE PREGUNTAS DENTRO } \\
\text { DEL INSTRUMENTO }\end{array}$ & 13 \\
\hline TIPO DE RESPUESTA & Escala likert \\
\hline & Por puntaje: \\
CRITERIO DE CALIFICACIÓN & 4 el aspecto se cumple en el modelo. \\
& De $3-3.9$ el aspecto se cumple en el modelo pero debe ser ajustado. \\
De $1-2.9$ el aspecto evaluado no se cumple dentro del modelo. \\
\hline FORMA DE LA EVALUACIÓN & Se suministró a cada evaluador el cuestionario de evaluación, la representación gráfica, \\
\hline COEFICIENTE A OBTENIDO & 0.87 \\
\hline
\end{tabular}

Fuente: elaboración propia.

\section{RESULTADOS Y DISCUSIÓN}

\section{Resultados de la recolección de información y la triangulación}

Se encontró que en los LIU visitados la mayoría aplica los elementos verificados dentro de la herramienta de observación que corresponden a requisitos establecidos dentro de los sistemas de gestión empleados como base para construir la herramienta (ISO 9001 e ISO-IEC 17025), excepto para la verificación por terceros de la buena ejecución de las actividades en el laboratorio.

Los resultados de las encuestas indican que algunos de los aspectos indagados entre los investigadores son aceptados como elementos que podrían incluirse dentro del modelo al obtener calificaciones entre 3.9 a 4 (20 de los 25 aspectos indagados). Sin embargo la mayoría de las respuestas apuntan a que debería buscarse otra fuente para concluir de manera definitiva si se incluían o descartaban dentro de la construcción del modelo (Figura 2.).

Fiǵura 2. Resultados aplicación de la encuesta

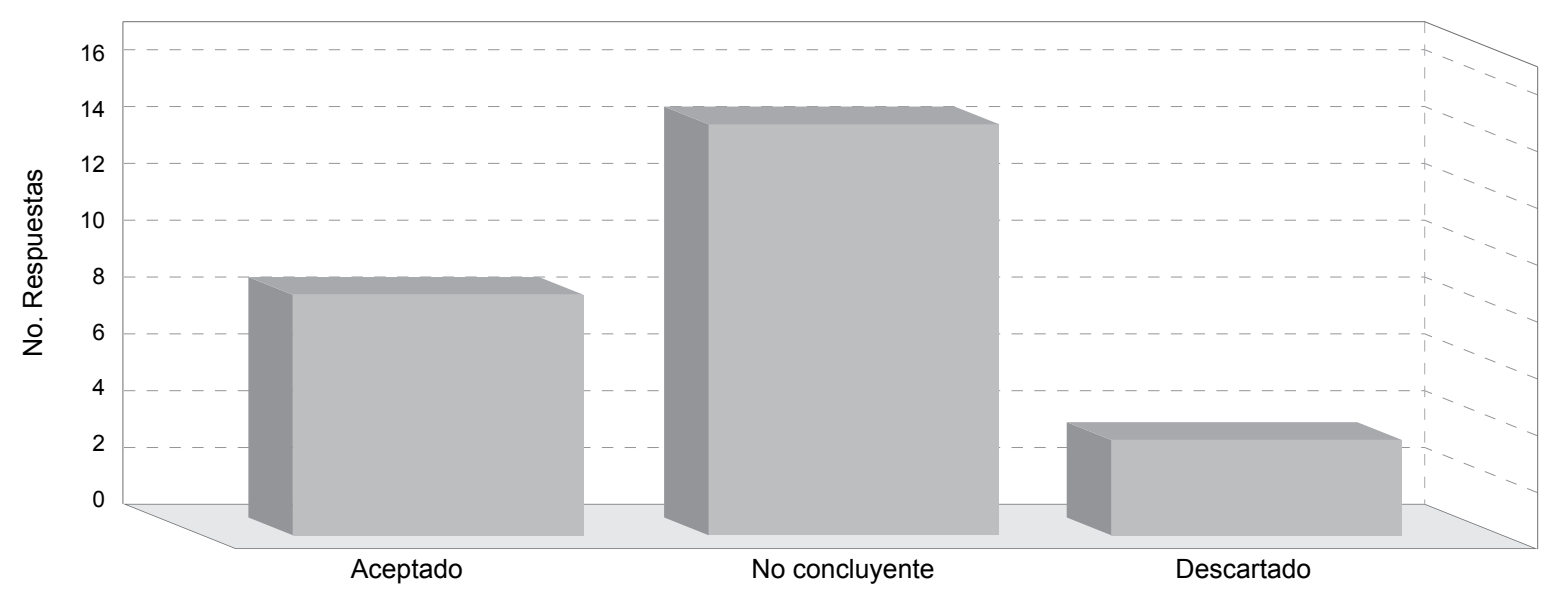

Fuente: elaboración propia. 
Dentro del análisis documental de las fuentes bibliográficas se encuentran elementos referentes a los laboratorios de investigación, que podrín ser incluídos en el modelo, tales como: el aseguramiento de calidad de los resultados obtenidos (trazabilidad, validez, equipos, condiciones ambientales, control de calidad), la documentación, la cultura organizacional dentro del equipo de investigación y el reconocimiento de los intereses de quienes realizan actividades de investigación. Así mismo, se deberían tener en cuenta las exigencias de los patrocinadores de las investigaciones que piden realizar reportes financieros o hacen mención al control del uso de los recursos invertidos en las investigaciones. También se hallaron exigencias legales para ciertas actividades de investigación como la gestión de residuos generados por el laboratorio, el manejo de animales en investigación y el acceso a material genético de especies silvestres. La inclusión de los aspectos encontrados en la revisión del marco teórico dentro de la triangulación amplió la extensión de varias conclusiones preliminares obtenidas a partir de los resultados de la aplicación de la encuesta y la herramienta de observación. Esto permitió definir la inclusión o no de aquellos aspectos que en las encuestas resultaron de manera preliminar como no concluyentes.

Otro resultado de la triangulación fue el hallar elementos contradictorios entre el marco teórico y los resultados de la encuesta que fueron descartados, en temas relacionados con aspectos ambientales y financieros. También se encontró que las investigaciones pueden tener un interés social debido a sus posibles aplicaciones, este interés recae en la comunidad no científica (que no es una parte interesada directamente relacionada a los laboratorios de investigación).

Por último, para el principio de relaciones mutuamente beneficiosas con el proveedor no se encontraron elementos en la revisión documental que pudieran emplearse dentro de la triangulación. La misma situación se presentó para la evaluación por terceros del laboratorio (auditoria).

\section{Resultados de la construcción del modelo}

El modelo se estructuró desde lo particular a lo general, de la siguiente manera: requisitos, título general de los requisitos y su organización por componente articulador (Tabla 6). Teniendo en cuenta que dentro de los aspectos encontrados en la identificación de la problemática de investigación fue la percepción frente a la poca aplicabilidad de las normas existentes, secomplementó el planteamiento de cada requisito propuesto con algunas recomendaciones deimplementación (como se ejemplifica en la Tabla 7) y un esquema de implementación general de los requisitos.

Tabla 6. Requisitos del modelo de gestión integral

\begin{tabular}{|c|c|c|}
\hline COMPONENTE ARTICULADOR & PRINCIPIO & REQUISITOS (GENERALIDADES) \\
\hline \multirow{2}{*}{ HUMANO } & \multirow{2}{*}{ Participación del personal } & Equipo de investigación \\
\hline & & Mejora en las actividades de investigación \\
\hline \multirow{8}{*}{ OPERATIVO } & \multirow{3}{*}{ Enfoque de sistema para la gestión } & Política y objetivos \\
\hline & & Infraestructura \\
\hline & & Documentación e información \\
\hline & \multirow{3}{*}{ Basarse en datos y hechos para tomar decisiones } & Ítem de estudio \\
\hline & & Equipos \\
\hline & & Búsqueda y procesamiento de información \\
\hline & Enfoque por procesos & $\begin{array}{l}\text { Aseguramiento de la calidad de las actividades } \\
\text { de investigación }\end{array}$ \\
\hline & Relaciones de beneficio mutuo con proveedores & Proveedores \\
\hline \multirow{3}{*}{ ESTRATÉGICO } & Liderazgo & Dirección del laboratorio \\
\hline & \multirow{2}{*}{ Enfoque al cliente } & Capacidad del laboratorio \\
\hline & & Clientes internos y externos \\
\hline
\end{tabular}

Fuente: elaboración propia. 
Andrea del Pilar García Pérez, William Javier Cepeda Páez

Tabla 7. Requisito propuesto y recomendaciones para su implementación

\begin{tabular}{|l|l|}
\hline \multicolumn{1}{|c|}{ REQUISITO: ÍTEM DE ESTUDIO } & \multicolumn{1}{|c|}{ RECOMENDACIONES PARA LA IMPLEMENTACIÓN } \\
\hline \multirow{2}{*}{$\begin{array}{l}\text { Fijar parámetros para el manejo adecuado } \\
\text { de los elementos de estudio. }\end{array}$} & $\begin{array}{l}\text { Se deben establecer dentro del laboratorio criterios de identificación de las muestras, } \\
\text { parámetros de control y la disponibilidad de instrumentos de medición para las áreas } \\
\text { de almacenamiento (temperatura, humedad, presión, etc.). } \\
\text { Deberían fijarse tiempos de almacenamiento de las muestras, procedimientos para evitar } \\
\text { su pérdida o confusión con otras muestras. }\end{array}$ \\
\hline
\end{tabular}

Fuente: elaboración propia.

- La construcción del modelo culminó con la elaboración de una representación gráfica que describiera las relaciones entre los principios, los componentes de articulación, el ciclo PHVA (planear, hacer, verificar y actuar) y las partes interesadas, dentro de un esquema que mostrara un sistema abierto característico de los SGI.

\section{RESULTADOS}

\section{DE LA PREVALIDACIÓN}

El primer resultado de la prevalidación fue no poderse refutar ni comprobar si el modelo permite asegurar la confiabilidad de los resultados de los LIU, debido a que los resultados obtenidos de la revisión hechos por los evaluadores sobre este aspecto resultó no concluyente. El resultado de las calificaciones dadas por los evaluadores fue el mismo para todos los aspectos revisados del modelo. Se cumplen todos los aspectos pero el modelo debe ser ajustado.

Los ajustes requeridos por el modelo, identificados dentro de la prevalidación, fueron los siguientes: no se identifica claramente el alcance del modelo ya que existe confusión entre el laboratorio, el proyecto de investigación y el grupo de investigación. Es necesario profundizar en el detalle de requisitos y recomendaciones de implementación teniendo en cuenta que los investigadores no manejan los términos de gestión de calidad. Así mismo, se necesita tener en cuenta que el director del proyecto cuenta con varias responsabilidades y las relaciones dentro de la representación gráfica propuesta no son muy claras. Solo uno de los nueve evaluadores consultados indicó que recomendaba la inclusión de la evaluación externa dentro del modelo.

De la aplicación de las evaluaciones realizadas dentro de la revalidación los ajustes realizados modificaron el modelo inicialmente propuesto, en el cual se incluyó un pequeño glosario a modo de orientación, se reorganizaron las actividades e incluyeron las actividades de planeación financiera. En el despliegue de los requisitos se llevó a cabo la ampliación en el detalle de los requisitos y de las recomendaciones de implementación. Para el caso de la representación gráfica se hizo una propuesta adaptada a los laboratorios en la cual se remplazaron los principios de ISO 9001 por los de un SGI y se remplazó el término de componente operativo por el de componente científico-técnico (figura 3). Estos dos últimos cambios se realizaron para mejorar la interpretación del lector con respecto a las relaciones generales de las partes que conforman el modelo. 
Figura 3. Representación gráfica del modelo para los LIU

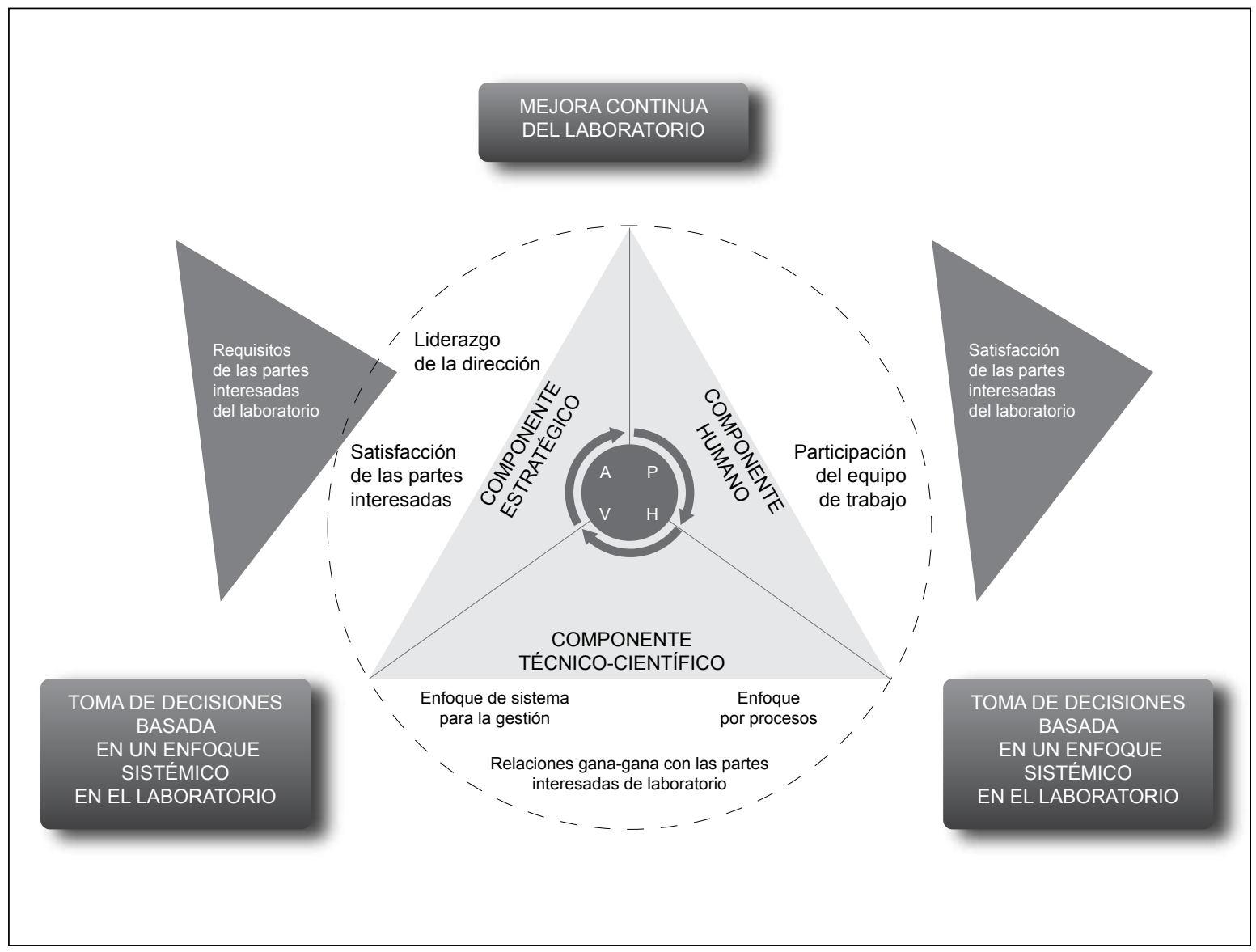

Fuente: elaboración propia

\section{CONCLUSIÓN}

La propuesta para un modelo de sistema de gestión integrado (SGI) para los laboratorios de investigación universitarios presenta un enfoque marcadamente operativo (componente operativo), no solo porque éste es el que más principios relaciona dentro del modelo sino porque también reúne a la mayoría de los requisitos (16 de 35) dentro del mismo, lo cual se considera propio de un sistema enfocado a un tipo de organización como los laboratorios.

Partir de un elemento universal y general como son los principios definidos dentro de la norma ISO 9000 permite la integración de normas existentes y también su articulación con otros enfoques sistémicos, como es el caso del sistema de gestión integrado. Sin embargo, debido a que para este último los principios que lo definen presentan un alcance más amplio, emplear normas definidas bajo el enfoque ISO no es suficiente para abarcar el alcance de un sistema de gestión integrado.

El modelo puede ser implementado en un laboratorio de investigación universitario, teniendo en cuenta los resultados de aceptabilidad presentados dentro de la prevalidación y lo encontrado en la recopilación de información preliminar en los laboratorios. Así mismo, estableciendo una estrategia de acercamiento previa a la implementación, teniendo en cuenta el poco conocimiento de los investigadores sobre los sistemas de gestión y la imagen negativa que se tiene en el ámbito científico de lo que implica implementar un sistema. 
Para lograr propuestas significativas, la integración del marco teórico con los resultados obtenidos a partir de encuestas o revisiones in situ es estratégico. En especial para la formulación de modelos basados en el enfoque de los sistemas de gestión integral, ya que no existen referencias normativas ni de gestión muy desarrolladas que puedan servir como base teórica.

Los componentes de articulación en el modelo permiten no solo evidenciar la relación entre los principios y las demás partes que describen el modelo de gestión; también evidencian las relaciones entre las partes del sistema y los requisitos, sin que se deba pasar por los principios del sistema.

La relación entre los laboratorios con sus proveedores de productos y servicios, así como con auditores y evaluadores no se incluyeron dentro del modelo debido a que a los resultados encontrados dentro de la indagación preliminar en los laboratorios de investigación, la revisión documental y la prevalidación no mostraron que fueran de importancia para este tipo de organizaciones.

\section{REFERENCIAS}

ALONSO MIGUEL, Pedro. Calidad en Investigación ( $1^{\mathrm{a}}$ y $2^{\mathrm{a}}$ parte). De qué trata la gestión de calidad en investigación. Aula Abierta [en línea] Diciembre, 2005. No. 32 y 33. Disponible en: http://www. madrimasd.org/revista/revista32/aula/aula1.asp

ARANGO SERNA, Martín Darío, LONDOÑO, Jesús Enrique y ZAPATA

CORTÉS, Julián Andrés. Arquitectura Empresarial- Una visión general. En: Revista de Ingenierías. Universidad de Medellín. 2010. Vol. 9, No. 16. p. 110-111. ISSN 1692-3324.
CABRERA CISTERNA, Francisco. Categorización y triangulación como proceso de validación del conocimiento en investigación cualitativa. Ensayo. En: Theoria, 2005. Vol. 14. p. 61-71. ISSN 0717-196X

CAMPO ARIAS, Adalberto y OVIEDO, Heidi [en línea]. Propiedades psicométricas de una escala la consistencia interna. En: Revista Salud Pública, Universidad Nacional de Colombia. 2008. Vol 5, No. 10. p. 831-839. ISSN 0124-0064. Disponible en: http://www.scielosp.org/pdf/rsap/v10n5/ v10n5a15.pdf

CERVANTES, Víctor H. [en línea]. Interpretaciones del coeficiente de alpha de Cronbach. En: Avances en Medición, Universidad Nacional de Colombia. 2005. Vol 3, No. 1. p. 9-28. ISSN 1692-0023. Disponible en: https://scienti.colciencias.gov.co:8084/ publidex/docs/artículos/1692-0023/2/1.pdf

\section{GESTIÓN DE CALIDAD EN LA INVESTIGACIÓN} [en línea]. Red iris. Disponible en: http://www. rediris.es/list/info/calidadinv.html

GLIEM, Joseph A y GLIEM, Rosemary R [en línea]. Calculating, interpreting, and reporting Cronbach's alpha reliability coefficient for Likert-type scales. Midwest Research-to-Practice Conference. En: Adult, Continuing, and Community Education. The Ohio State University. 2003. p. 82-88. Disponible en: https://scholarworks.iupui.edu/handle /1805/344

VOM BROCKE, Jan y ROSEMAN, Michael. Handbook of Business Process Management 1. 2010. Berlin: Springer-Verlag. 623 p. ISBN 978-3-642-00415-5

JOINT IAF-ILAG-ISO COMMUNIQUÉ ON THE MANAGEMENT SYSTEMS REQUIREMENTS OF ISO/IEC 17025:2005 [en línea]. General requirements for the competence of testing and calibration Laboratory. January 2009 IAF, ILAC, 
ISO. Disponible en: http://www.nist.gov/nvlap/ upload/2009-01-08_IAF-ILAC-ISO-communiquC3-83-_ISO-IEC17025_-1.pdf

RESPONSIBLE CONDUCT IN THE GLOBAL RESEARCH ENTERPRISE [en línea]. A Policy Report. InterAcademy Council. Septiembre 2012. Disponible en: www.interacademycouncil.net. p. 15.

ROBINS, Margaret M, SCARLL, Jane S y E. KEY, Pauline. Quality assurance in research laboratories. Accred Qual Assur [en línea]. Springer-Verlag. Abril, 2006. p. 214 - 223. Disponible en: http:// www.springerlink.com/?MUD=MP
TEJADA LOSADA, Fabio y PEÑA GUARÍN, Guillermo. Reflexiones sobre las características constitutivas de la gestión integral. En: SIGNOS. Vol.1, No. 2, 2009. p. 79-93, ISSN 2145-1389

VARAS, Marika, SÁNCHEZ, Luis y ALVARADO, Luis. Los directores de proyectos de investigación: competencias y desafíos. R\&D\&i Project Managers: professional competitions and challenges. Dirección y Organización, Diciembre, 2009. No. 39. Disponible en: http://www.revistadyo.com/index. php/dyo/article/viewFile/25/25. ISSN: 1132-175X

VELÁSQUEZ RUEDA, María del Rosario. Planificación de un sistema de gestión de la calidad como plataforma para integrar otros modelos de gestión. En: Signos. Vol. 4, No. 2, 2012. p. 15-31. 